\title{
Investigation on Antenna Coupling in Pulsed Arrays
}

\author{
Matteo Ciattaglia, Student Member, IEEE, and Gaetano Marrocco
}

\begin{abstract}
Pulsed arrays are becoming popular in new ultrawide-band applications to achieve long-range coverage, high capacity and to identify the angle of arrival of multiple echoes in complex environments. As in the monochromatic regime, distortion of ultrawide-band transmitted and received signals can result from the coupling between individual radiators that are in close proximity. This paper investigates the time-domain coupling in finite arrays that radiate short pulses, by introducing the concept of time-domain "active" array factor and "active" element factor. The proposed model highlights the signal-distortion phenomenology and obtains useful guidelines to reduce pulse coupling, even in dense arrays, by a proper choice of the geometrical and electrical parameters.
\end{abstract}

Index Terms-Active element factor, mutual coupling, pulsed arrays, Radon transforms, ultrawide-band antennas.

\section{INTRODUCTION}

I MPULSE-radiating arrays have been recently proposed for several ultrawide-band (UWB) applications [1] in radar, communications, remote sensing and indoor localization, exploiting their advantages in terms of reduced sidelobes and high resolution. Impulse-radiating arrays employ UWB antennas, which are driven by pulsed waveforms, and their scanning performances are controlled by inter-element time delays. Preliminary physical investigations have been reported in [2]-[4] where coupling between radiating elements is usually neglected and emphasis is mainly devoted to conditions for grating lobe cancellation, permitting the design of sparse arrays. The reduction in sidelobe levels is due to the combined angular and temporal spreading of the energy radiated by the antenna. The presence of the temporal dimension in the radiation phenomenology [4] plays an important role also in the coupling estimation, as it will be explained in this paper.

In the frequency domain, for the case of narrow-band antennas, the well-established theory of the active element factor [5] permitted to predict the malicious effect of antenna mismatch and scan blindness in phased arrays.

In the time domain, any impulse radiating antenna has to be considered as an angular-temporal filter, in the sense that it not only distributes the power along the observation angles, but it also introduces a signal distortion on the input pulse [6] depending on the antenna shape and on the observation angle. In

Manuscript received May 13, 2005; revised November 16, 2005.

M. Ciattaglia is with the Dipartimento di Informatica, Sistemi e Produzione, University of Roma "Tor Vergata," 00133 Roma, Italy. He is also with SELEX SI S.p.A., 00131 Rome, Italy.

G. Marrocco is with the Dipartimento di Informatica, Sistemi e Produzione, University of Roma "Tor Vergata," 00133 Roma, Italy (e-mail: marrocco@disp.uniroma2.it).

Digital Object Identifier 10.1109/TAP.2006.869930 array configurations, the latter distortion effect can be potentially emphasized at some scanning direction and according to the duration of the input signal.

Fullwave models of real TD arrays including coupling have been recently proposed [7], [8] for the particular case of infinite periodic geometries where the inter-antenna interaction is directly taken into account by the excitation of time-domain Floquet waves. In finite arrays, like those proposed for ultrawide-band applications in complex scenarios, the Floquet-waves framework does not apply and the time domain coupling phenomenology is hidden in the overall system response.

This paper proposes a simple physical model of the time domain coupling for impulse-radiating finite arrays aimed to identify the role of the scan angle, the input signal duration, the repetition rate of the input pulse train and finally the impulse response of the single antenna. Expressions for the time domain (TD) effective height and effective array factor are retrieved, and an investigation on coupling echoes and their distorting effect on the main signal permits to obtain conditions to reduce coupling, even in compact configurations with very small inter-element distances. The paper is organized as follows: Section II recalls the basic definitions of antenna impulse responses and time-domain array factor operator, and new expressions for the TD active array factor and element factor are retrieved in Section III. Section IV presents a qualitative investigation on the TD coupling in pulsed arrays under some simplifying hypothesis, while Section V shows some results for a realistic fullwave example.

\section{BASIC DEFINITIONS: IMPULSE RESPONSE OPERATOR AND TD ARRAY FACTOR}

The antenna model of Fig. 1(a) is considered for the following discussion. The antenna input port is connected, through a transmission line of characteristic impedance $R_{0}$, to a real voltage source $v_{i n}(t)$ with internal resistance $R_{g}=R_{0}$. The time-dependent port variables are the TD reflection coefficient $\gamma_{a}(t)$ or, alternatively, the TD input impedance or admittance $z_{a}(t), y_{a}(t)$. These functions have the meaning of input impulse responses working as convolution operators on nodal or on travelling wave functions such as: $v^{-}(t)=\gamma_{a}(t) * v^{+}(t)$, or $v(t)=z_{a}(t) * i(t)$, where ${ }^{*} *$, denotes convolution, $v^{+}(t)=$ $(1 / 2) v_{i n}\left(t-t_{g}\right)$ and $v^{-}(t)$ are the travelling inward and outward voltage waves, $t_{g}$ is the time delay along the line (supposed zero, for simplicity) and $v(t), i(t)$ are the antenna nodal voltage and current $\left(v=v^{+}+v^{-}, i=i^{+}+i^{-}, i^{+}(t)=\left(1 / R_{0}\right) v^{+}\right)$. Time-dependent radiation is generally described by the transmitting-mode TD effective height [6] $\boldsymbol{h}^{t}(\hat{\boldsymbol{r}}, t)$ which accounts for the antenna-source mismatch. 


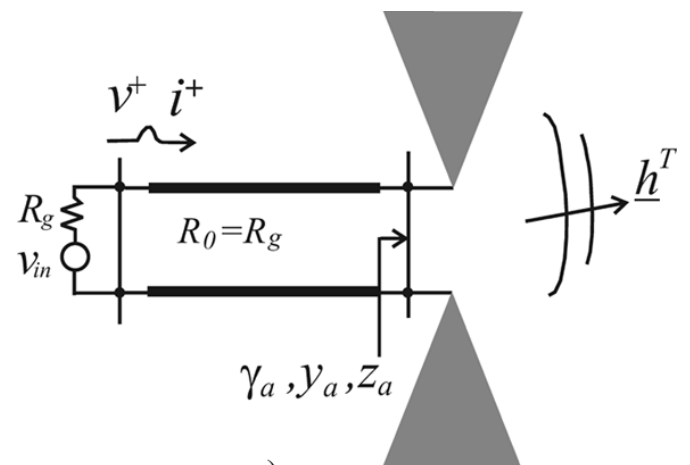

a)

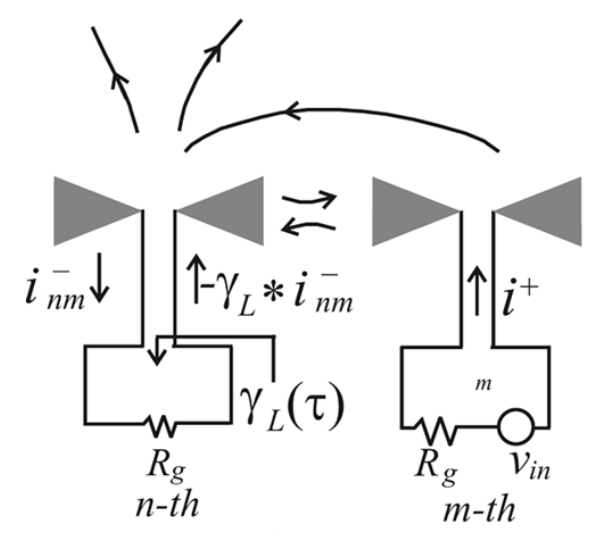

b)

Fig. 1. (a) Time-dependent antenna input and radiation functions; (b) Current wave components at the $n$th antenna port due to the coupling with the $i_{m}^{+}(t)$ current at the $m$ th antenna.

A set of $N$ identical antennas, each excited by an inward travelling current $i_{n}^{+}(\tau)$ is now considered. Following usual far-field approximations, the total field radiated by the array, when the coupling effects are neglected $\left(\boldsymbol{h}_{n}^{t}=h^{t}\right)$ is

$$
\boldsymbol{E}^{A}(\boldsymbol{r}, t)=-\frac{\eta_{0}}{4 \pi r c}\left[\boldsymbol{h}^{t}(\hat{\boldsymbol{r}}, \cdot) * \mathcal{F}(\hat{\boldsymbol{r}}, \cdot)\right]\left(t-\frac{r}{c}\right)
$$

where $\mathcal{F}(\hat{\boldsymbol{r}}, \tau)=\sum_{n=1}^{N} i_{n}^{+}\left(\tau+t_{n}(\hat{\boldsymbol{r}})\right)$ is the TD array factor with $t_{n}(\hat{\boldsymbol{r}})=\left(\boldsymbol{r}_{n} \cdot \hat{\boldsymbol{r}}\right) / c$ and $\boldsymbol{r}_{n}$ tags the $m$ th antenna position [2].

\section{TD ACTIVE ARRAY FACTOR AND ACTIVE ELEMENT FACTOR}

The conventional array coupling model in the frequency domain [5] needs to be properly modified to obtain the time-dependent coupling scheme. The above model requires the total port current, e.g., the superposition of direct and reflected currents, and supposes a perfect matching between the antenna and the source. This last condition, as discussed in [6], needs to be dropped when considering an UWB antenna since the multiple reflections between antennas and loads are already embedded into the TD effective height. Therefore, with reference to Fig. 1(b), the total nodal port current $i_{T, n}(t)$ at the $n$th antenna, when coupled with all the others, is

$$
\begin{aligned}
i_{T, n}(t)=i_{n}^{+}(t)-\gamma_{a, n}(t) * i_{n}^{+}(t) & \\
& +\sum_{\substack{m=1 \\
m \neq n}}^{N}\left[i_{n m}^{-}(t)-\gamma_{L, n}(t) * i_{n m}^{-}(t)\right]
\end{aligned}
$$

where $\gamma_{a, n}(\tau)$ is the embedded time-dependent reflection coefficient operator of the $n$th antenna accounting for the presence of the passive external environment, e.g., of other antennas, each connected to its own load. $\gamma_{L, n}(\tau)$ is the embedded reflection coefficient of the same antenna but looking toward voltage source $\left(\gamma_{L, n}=-\gamma_{a, n}\right.$, if the source is directly connected to the antenna); $i_{n m}^{-}(t)$ is the received outward-traveling current at $n$th antenna port (from the antenna to the load) due to the $m$ th antenna direct current wave $i_{m}^{+}(t)$, under the hypothesis that the antenna is terminated by a matched load corresponding to the embedded input impedance $z_{L}=z_{a, n}(t)$. By linearity, outward coupling currents $i_{n m}^{-}(t)$ can be related to the source currents $i_{m}^{+}(t)$ at the $m$ th antenna by the convolution integral $i_{n m}^{-}(t)=-s_{n m}(t) * i_{m}^{+}(t)$, when all the antennas, other than the $m$ th, are connected to the internal load $R_{g}$ of their source. The function $s_{n m}(\tau)$ is a time-dependent coupling operator which accounts for the multiple bouncing among radiators under perfect matching condition of the "victim" ( $m$ th) antenna. The antenna effective height operator needs to be applied only to total inward waves $i_{T, n}^{+}=i_{n}^{+}-\gamma_{L, n} * \sum_{\substack{m=1 \\ m \neq n}}^{N} i_{n m}^{-}$. The resulting time-dependent active array factor, including inter-antenna coupling, is therefore

$$
\begin{aligned}
\mathcal{F}_{A}(\hat{\boldsymbol{r}}, \tau)= & \sum_{n=1}^{N} i_{n}^{+}\left(\tau+t_{n}(\hat{\boldsymbol{r}})\right) \\
& -\sum_{n=1}^{N} \gamma_{L, n}(\tau) * \sum_{\substack{m=1 \\
m \neq n}}^{N} s_{n m} * i_{m}^{+}\left(\tau+t_{n}(\hat{\boldsymbol{r}})\right)
\end{aligned}
$$

The last summation shows the spatial and temporal distortion of the input signals due to the coupling among antennas. Note that this expression differs from the corresponding frequency domain function for the presence of the $\gamma_{L, n}(\tau)$ operator and since it applies to travelling waves rather than to nodal functions.

The field radiated by the array, when only the $k$ th element is driven, is obtained from (3) and (1) as

$$
\boldsymbol{E}_{k}(\boldsymbol{r}, t)=-\frac{\eta_{0}}{4 \pi r c}\left[\boldsymbol{h}_{k}^{t}(\hat{\boldsymbol{r}}, \cdot) * i_{k}^{+}(\cdot)\right]\left(t-\frac{r}{c}\right)
$$

where $\boldsymbol{h}_{k}^{t}$ is the TD active effective height of the $k$ th radiating element

$$
\begin{aligned}
& \boldsymbol{h}_{k}^{t}(\hat{\boldsymbol{r}}, \tau)=h^{t}(\hat{\boldsymbol{r}}, \tau) \\
& \quad-\sum_{\substack{n=1 \\
n \neq k}}^{N} \gamma_{L, n}(\tau) * s_{n k}(\tau) * \boldsymbol{h}^{t}\left(\hat{\boldsymbol{r}}, \tau+t_{n}(\hat{\boldsymbol{r}})-t_{k}(\hat{\boldsymbol{r}})\right) .
\end{aligned}
$$

The distortion function, e.g., the summation in (3), depends on the source-antenna mismatch as well as on the mutual distances among radiators through the term $t_{n}(\hat{\boldsymbol{r}})-t_{k}(\hat{\boldsymbol{r}})$.

\section{INVESTIGATION ON COUPLING ECHOES}

Some qualitative information about the distortion effect and in particular about the role played by the scan direction, the pulse width, the pulse repetition rate and the array density, can be earned by introducing some simplifying hypothesis. With reference to (3), it is supposed a regular alignment of antennas along $\hat{x}$ axis with inter-element spacing $d$. The sourcing currents are trains of equi-amplitude delayed waveforms $g(t)$, e.g., 
$i_{n}^{+}(\tau)=g(t) * \sum_{l=1}^{N_{L}} \delta\left(\tau-n t_{s}-l T_{p}\right)$ where $T_{p}$ is the repetition rate and $t_{s}=(d / c) \sin \theta_{0}$ a linear delay to achieve beam scanning along $\theta_{0}$. It can be convenient to further suppose that:

i) the coupling factors $s_{n m}$, which can not be generally expressed into analytical forms, may be approximated by the expression $s_{m n}(\tau) \sim S_{0}\left(\delta\left(\tau-r_{n m} / c\right) / r_{n m}\right)$ where $r_{n m}=\left|r_{n}-r_{m}\right|$ is the distance between elements and $S_{0}$ a constant factor. It is therefore assumed that the coupling current $i_{n m}^{-}$on the $n$th antenna is an undistorted shifted replica of the driving current on the $m$ th antenna which is attenuated according to the far field rule $1 / r_{n m}$;

ii) each antenna has the same embedded reflection coefficient. This is reasonably true for large arrays. Additionally, this function is supposed to be frequency independent, at least within the band of interest, e.g., $\gamma_{L, n}(\tau)=\Gamma_{o} \delta(\tau)$. It will be shown in the Example section that such simplified assumptions however permit to model the response of real arrays.

\section{A. Coupling for Dirac Pulse Input Signals}

Within the additional assumption that the driving signal is a Dirac pulse, e.g., $g(t)=\delta(t)$, the active array factor becomes

$$
\begin{aligned}
\mathcal{F}_{A}(\hat{\boldsymbol{r}}, \tau)= & \sum_{n=1}^{N} \sum_{l=1}^{N_{L}} \delta\left(\tau+t_{n}-n t_{s}-l T_{p}\right)-\sum_{n=1}^{N} \sum_{\substack{m=1 \\
m \neq n}}^{N} \frac{G_{0}}{|n-m|} \\
& \times \sum_{\ell=1}^{N_{L}} \delta\left(\tau+t_{n}-m t_{s}-\ell T_{p}-|n-m| \frac{d}{c}\right)
\end{aligned}
$$

where $G_{0}=S_{0} \Gamma_{0} / d$. The second summation accounts for the echoes generated by the coupling, e.g., by antenna diffractions. Echoes' delays depend on observation angle, element position, scanning direction and pulse position in the train, and they are superimposed to the direct pulses (first summation).

The above formula could be useful to predict a real coupling when the parameters $S_{0} / d$ and $\Gamma_{0}$, and hence $G_{0}$, are retrieved as average values from measurements or from antenna simulations. To emphasize the visualization of the echoes, the parameter $G_{0}$ is now set to 1 . A more realistic value will be considered in the next section. Within this assumption, and for the case of $N=4$ elements, $N_{L}=1$ and inter-element spacing $d=4 \mathrm{~cm}$, Fig. 2 shows the arrival time of the direct pulses and of the echoes for some scanning angles and different observation directions. It is possible to see that echoes emerging from different antennas may be partially superimposed, depending on the scan angle and observation point.

In particular, along the main lobe $t_{n}\left(\theta_{0}\right)=n t_{s}$ and therefore

$$
\begin{aligned}
& \mathcal{F}_{A}(\hat{\boldsymbol{r}}, \tau)=N \delta(\tau) \\
& \quad-\sum_{n=1}^{N} \sum_{\substack{m=1 \\
m \neq n}}^{N} \frac{G_{0}}{|n-m|} \delta\left(\tau+\left[(n-m) \sin \theta_{0}-|n-m|\right] \frac{d}{c}\right)
\end{aligned}
$$

It can be proved that the earliest echo in the scan direction is emitted at time

$$
t_{e, 1}=\frac{\left(1-\left|\sin \theta_{0}\right|\right) d}{c} .
$$
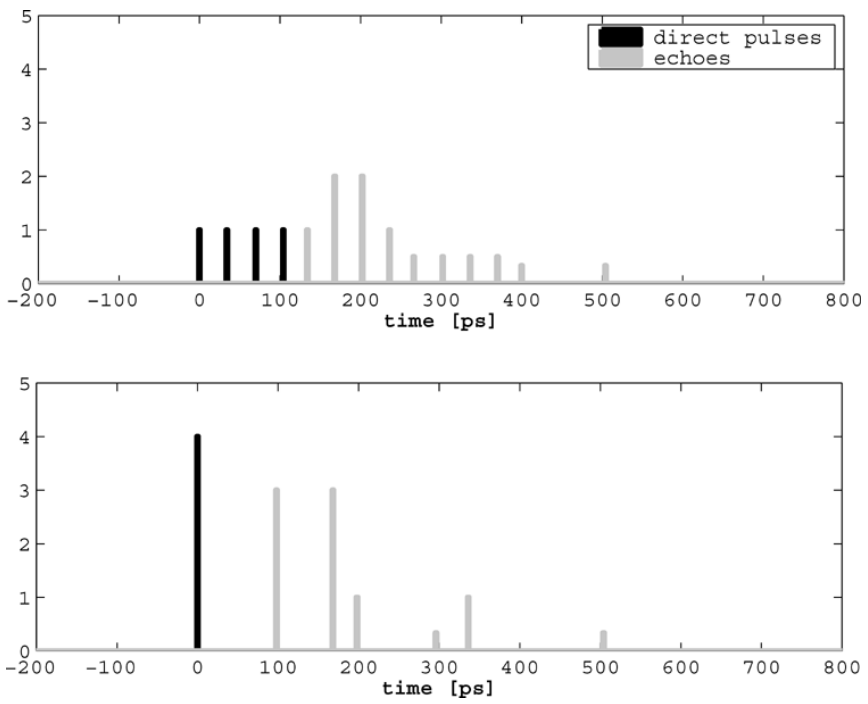

Fig. 2. Delay time of the direct pulses and of the coupling echoes for a $N=4$ element array with spacing $d=4 \mathrm{~cm}$, main lobe in $\theta_{0}=15^{\circ}$ and observation in $\theta=0^{\circ}$ (up) and $\theta=15^{\circ}$ (bottom). It has been assumed $G_{0}=1$. In this latter graph, different echoes appear at a same time.

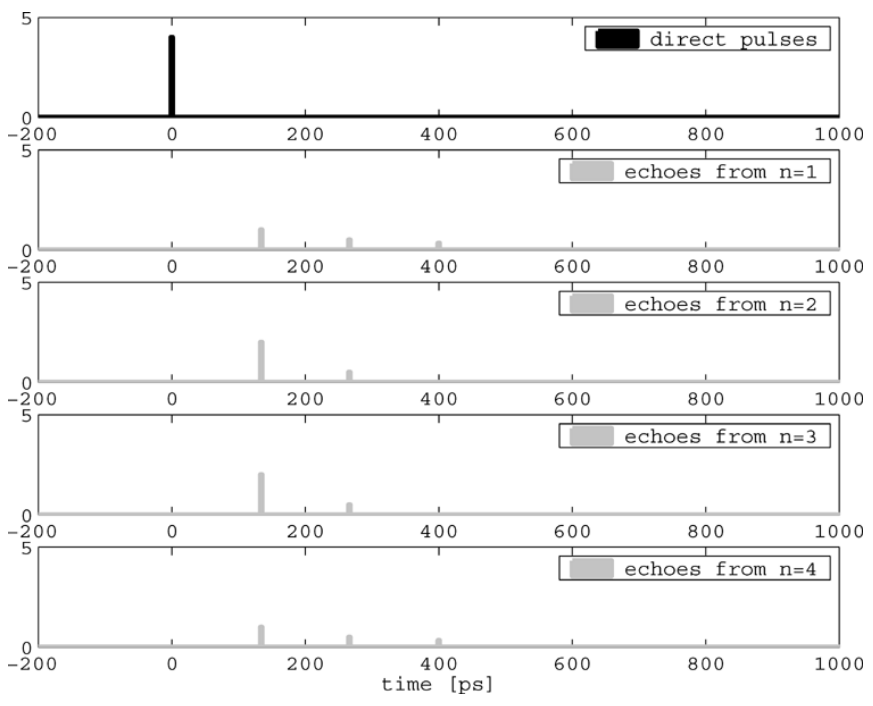

Fig. 3. Delay time of the coupling echoes along the main lobe oriented at $\theta_{0}=$ $0^{\circ}$ for an array of $N=4$ elements and spacing $d=4 \mathrm{~cm}$ and $G_{0}=1$.

Fig. 3 and Fig. 4 display the arrival time of the echoes with respect to the direct pulse along the main beam at two different angles. In accordance to (7), as the scan angle approaches the endfire direction, the delay of the first echoes reduces to zero. In that case the first echoes and the direct pulses are fully synchronized and the coupling may produce sensible distortion to the radiated signal.

If $N_{L}>1$, e.g., in the case of multiple transmitting pulses, an incoming signal belonging to the direct pulse train can be distorted by the coupling contributions of a previous pulse ("cross-pulse coupling") even for a scanning angle different from the endfire. In [2], spurious lobes (cross-pulse lobes) caused by "pulse interference" (in contrast with canonical sidelobes caused by "phase interference") are examined and the condition for their presence, $d / T_{p} \gg c$, was retrieved. As in [2], sourcing signals with high pulse repetition rates or 


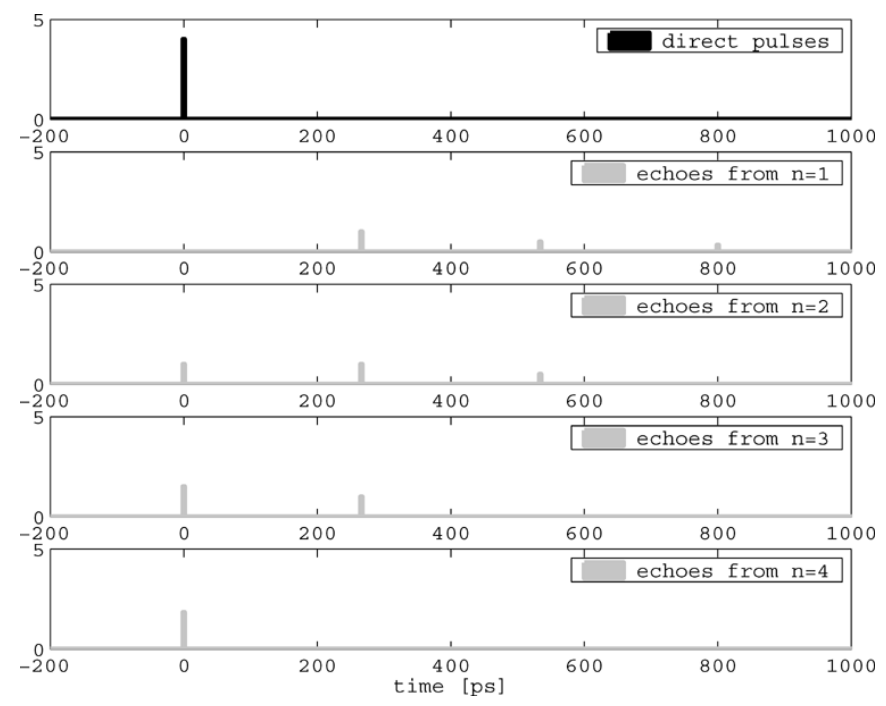

Fig. 4. Delay time of the coupling echoes along the main lobe oriented at $\theta_{0}=$ $90^{\circ}$ for an array of $N=4$ elements and spacing $d=4 \mathrm{~cm}$ and $G_{0}=1$.

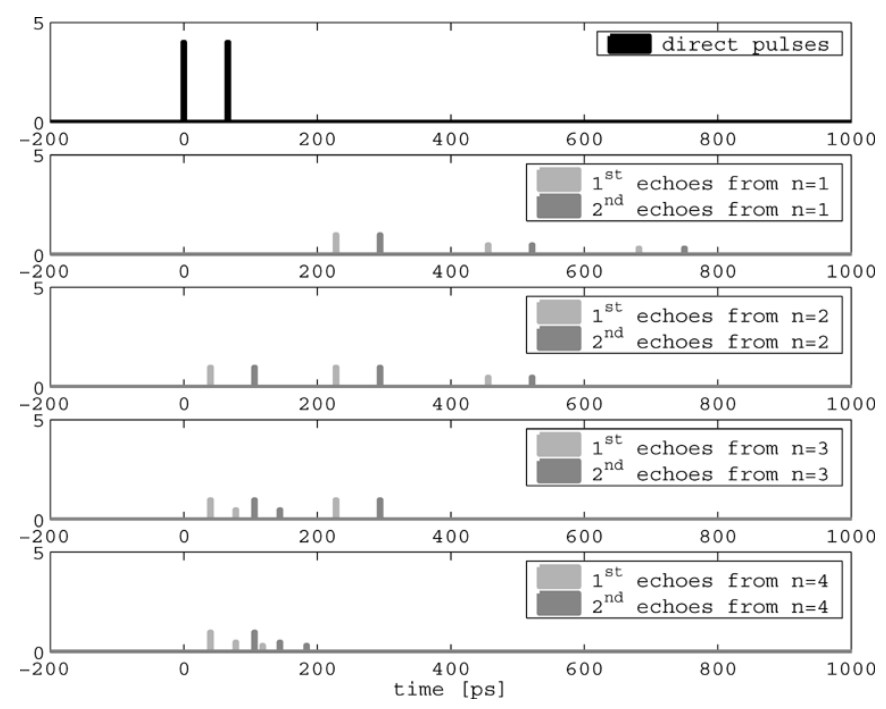

Fig. 5. Delay time of the coupling echoes along the main lobe oriented at $\theta_{0}=$ $45^{\circ}$ for an array of $N=4$ elements and spacing $d=4 \mathrm{~cm}, G_{0}=1$. The input signal is a train of $N_{L}=2$ pulses with repetition rate $d / T_{p}=2 c$. It is clearly visible how the first echoes, e.g., those originated by $\ell=1$ direct pulses are nearly superimposed to the $\ell=2$ direct pulses. Observation angle at $\theta=45^{\circ}$.

arrays with sparse configurations can potentially produce interferences between the $\ell^{\prime}$ th direct pulse and echoes originated from $\left(\ell<\ell^{\prime}\right)$ th direct pulses. For instance Fig. 5, referring to $N_{L}=2$ and $d / T_{p}=2 c$, shows how the echoes generated by the $\ell=1$ pulses tend to be superimposed to the $\ell=2$ direct pulses.

The condition on the pulse repetition rate which ensures the separation of the contributions of different pulses is

$$
T_{p}>\frac{L_{a}}{c\left(\sin \theta_{0}+1\right)}
$$

where $L_{a}$ is the array length. It can be seen again that the endfire scan is the most penalized one, since it requires the longest temporal separation, $T_{p}$, among pulses.

\section{B. Coupling for Finite-Duration Pulses}

A more realistic even still qualitative analysis can be performed by taking into account, rather than a Dirac pulse, an input waveform $g(t)$ of finite duration $T_{0}$. It is expected that the coupling strength will be also dependent on $T_{0}$ : if the pulse duration is longer than the time delay of the first echo, e.g., $T_{0}>t_{e, 1}$, direct pulses and echoes will be partially superimposed even at scan angles different from the endfire.

Additionally, to account for the limited bandwidth of real antennas, which sensibly affects the shape of the echoes and therefore the coupling mechanism, the time-domain effective height of the antenna is hereafter directly considered in the interference pattern of the array. The following simple mathematical model of the effective height, accounting for antenna distortion, is introduced in (5)

$$
\boldsymbol{h}^{t}(\hat{\boldsymbol{r}}, \tau)=H_{0}(\theta) \delta^{q}(\tau) \hat{\boldsymbol{p}}
$$

where $\delta^{q}(\tau)$ is the differential operator of $q$ th order, the factor $H_{0}$ is an angular-spreading factor and the only co-polar $\hat{\boldsymbol{p}}$ contribution to the radiated field is considered. For instance $q=2$, $H_{0}=-L^{2} \sin \theta /(2 c)$ and $\hat{\boldsymbol{p}}=\hat{\boldsymbol{\theta}}$ yield the time dependence of a short dipole of length $L$ as in [6]. According to this definition, and under the same hypothesis on mutual coupling of the previous section, the field radiated by the array of isotropic radiators $\left(H_{0}=1 \mathrm{~ms}^{n-1}\right)$ of order $n$ is

$$
\begin{aligned}
r E^{A}(\boldsymbol{r}, \tau) & \\
= & -\frac{\eta_{0}}{4 \pi r c} \sum_{n=1}^{N} \sum_{l=1}^{N_{L}} \partial_{\tau}^{q} g\left(\tau+t_{n}-n t_{s}-l T_{p}\right) \\
& -\sum_{n=1}^{N} \sum_{\substack{m=1 \\
m \neq n}}^{N} \frac{G_{0}}{|n-m|} \\
& \times \sum_{l=1}^{N_{L}} \partial_{\tau}^{q} g\left(\tau+t_{n}-m t_{s}-l T_{p}-|n-m| \frac{d}{c}\right)
\end{aligned}
$$

It is now considered an array of radiators of $(q=2)$-type excited by gaussian-pulse waveform $g(t)=\exp [-((t-$ $\left.\alpha)^{2}\right) / 2 \beta^{2}$, whose parameters $\alpha$ and $\beta$ are set to control its practical duration $T_{0}=\|t g(t)\|_{2} /\|g(t)\|_{2}$, where $\|\cdot\|_{2}$ is a $L_{2}$ norm. Hereafter, the radiated fields will be observed on the $\phi=0$ plane. Fig. 6 and Fig. 7 show the angle-time plot of the field radiated by an array of $N=4$ elements, with and without coupling, which have been timed to produce endfire radiation. It is here assumed a coupling factor $G_{0}=0.15$ which corresponds to reasonable values $\Gamma_{0}=-10 \mathrm{~dB}$ and $S_{0} / d=-6 \mathrm{~dB}$.

It can be noted the coherence point at $\theta=90^{\circ}$, and the four distinct contributions from the radiating elements at observation angles far from the boresight.

The effect of coupling between radiating elements is visible (see Fig. 7) as oscillations in the late transient of the radiated field, even in the main body of the waveform, since $T_{0}>t_{e, 1}$ $\left(T_{0}=0.3 \mathrm{~ns}\right.$ and $\left.t_{e, 1}=0\right)$.

The variation of the coupling with the scanning angle can be evaluated introducing a square error indicator which quantifies the effect of inter-element coupling versus the scanning angle and versus the duration $T_{0}$ of the input signal. The last one is 

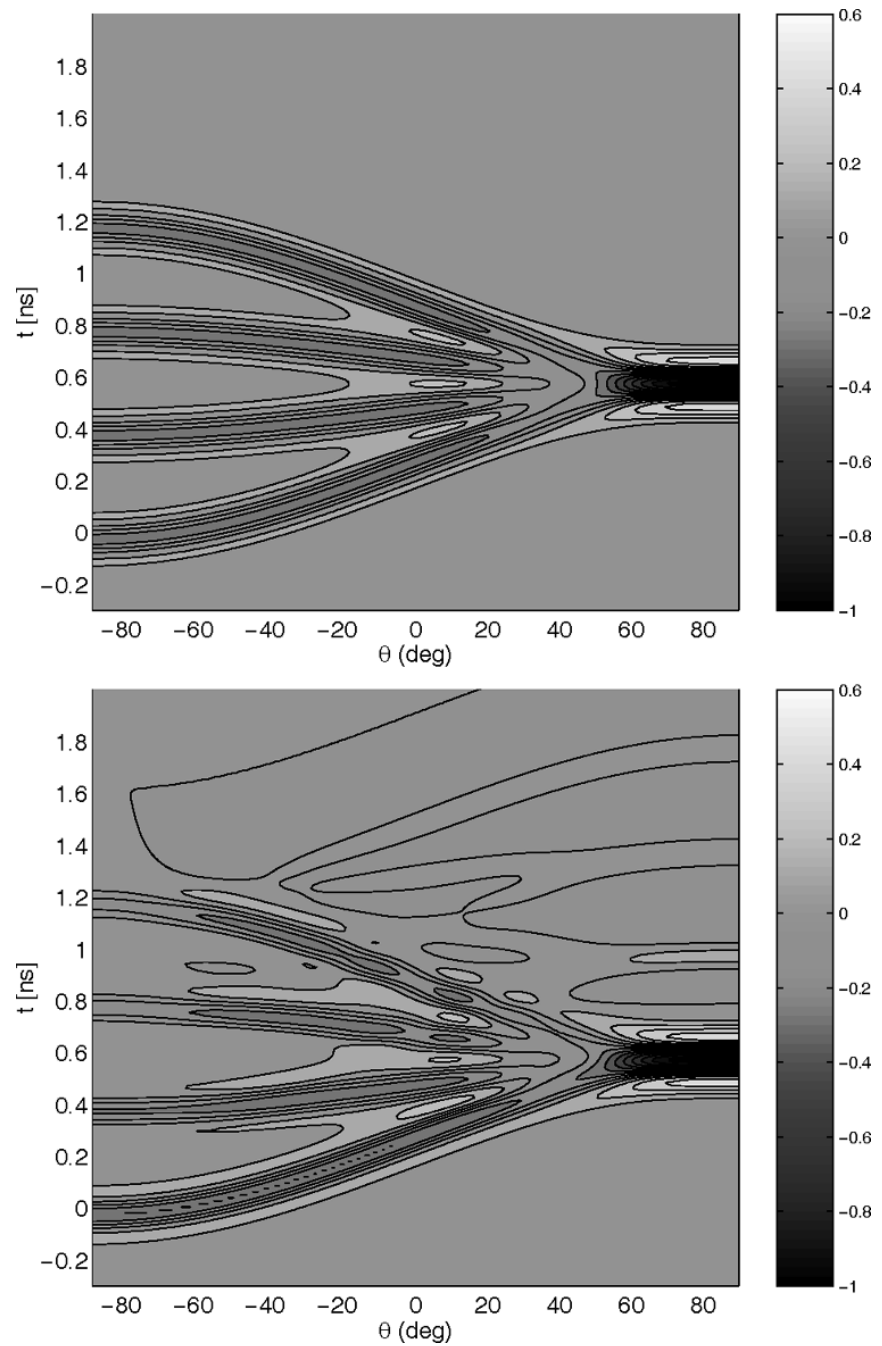

Fig. 6. Transient field with coupling (bottom) and without coupling (up) for an array of four isotropic radiating elements with $n=2, T_{0}=0.3 \mathrm{~ns}, N=4$, $d=6 \mathrm{~cm}, G_{0}=0.15, \phi=0^{\circ}$.
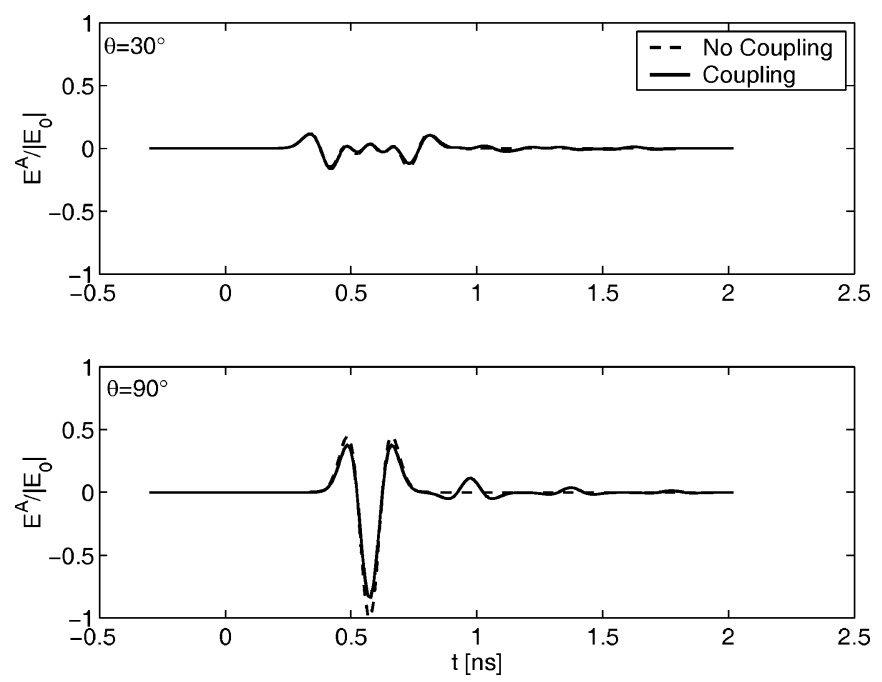

Fig. 7. Array fields from Fig. 6 at observation line $\theta=30^{\circ}$ (up) and $\theta=90^{\circ}$ (bottom), comparing the radiated dynamics with and without array coupling in the case of beam steering at $\theta_{0}=90^{\circ}$.

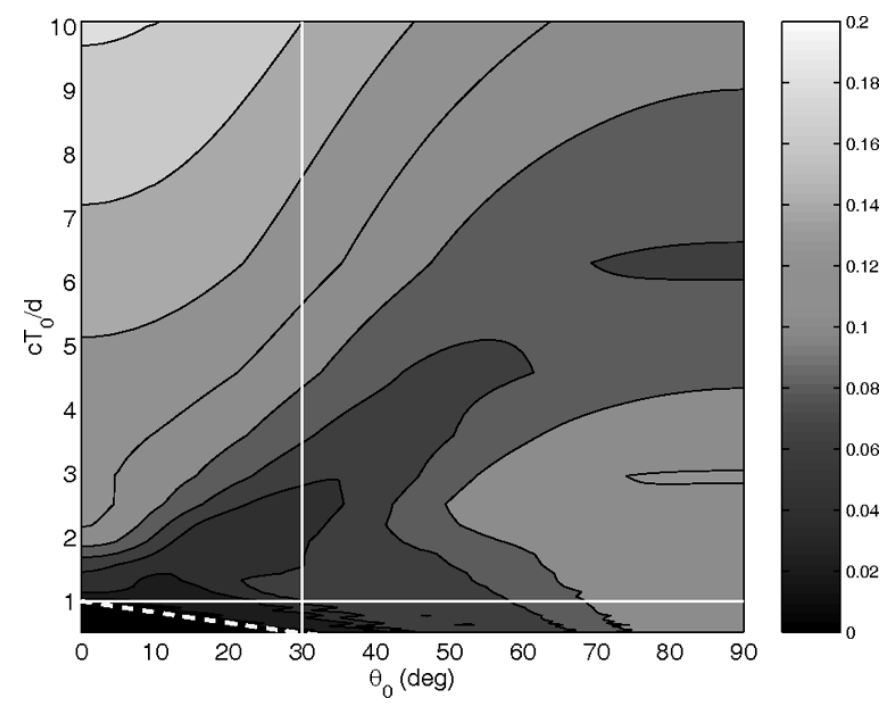

Fig. 8. Variation of the indicator $a\left(\theta_{0}, T_{0}\right)$ versus the scanning angle and the pulse duration for an array of $N=4$ isotropic elements with second order $(n=2)$ differentiation as time dependence.

considered within a temporal range which includes the main part of the radiated signal

$$
a\left(\theta_{0}, T_{0}\right)=\frac{\left\|\boldsymbol{E}_{c}^{A}\left(\theta=\theta_{0}, t, T_{0}\right)-\boldsymbol{E}_{n c}^{A}\left(\theta=\theta_{0}, t, T_{0}\right)\right\|_{2}}{\left\|\boldsymbol{E}_{n c}^{A}\left(\theta=\theta_{0}, t, T_{0}\right)\right\|_{2}}
$$

where the $L_{2}$ norm $\|\cdot\|_{2}$ is evaluated with respect to time for $t_{0}-T_{0} / 2<t<t_{0}+T_{0} / 2$ and $t_{0}$ is such that $\boldsymbol{E}_{c}^{A}\left(\theta_{0}, t_{0}\right)$ is the peak value. Only the main part of the signals is therefore considered.

The dashed line in Fig. 8 represents the relationship $T_{0}=t_{e, 1}$ e.g. $c T_{0} / d=\left(1-\left|\sin \theta_{0}\right|\right)$, deduced from (7), which separates the overlapping and nonoverlapping of the coupling echoes to direct pulses. In particular, for array density and duration of signals such that $c T_{0} / d<1$, the $a\left(\theta_{0}, T_{0}\right)$ indicator increases along with the scanning angle since in this case $t_{e, 1}$ reduces. Similarly, for scanning angles smaller than $30^{\circ}$ the indicator increases either with the signal duration $T_{0}$ or with the reduction of the array spacing. In both the cases, an increasing value of signal duration, array density or scanning angle leads to coupling contributions with greater distortion of the main signal.

In the region $c T_{0} / d>1$ and $\theta_{0}>30^{\circ}$, the monotonic behavior of the indicator with respect to the scanning angle and signal duration is generally lost.

Finally, it can be observed that the mostly relevant distortion of the direct waveform is produced for large-duration input signals in broadside arrays $\left(\theta_{0}=0^{\circ}\right)$. Because of the symmetry imposed by this scanning angle with respect to the array geometry, the coupling echoes produced by symmetric radiators are fully synchronized (see again Fig. 3) and therefore the strength of coupling echoes is reinforced.

For different scanning directions, instead, mutual coupling contributions produced from radiator pairs lose their temporal synchronization and spread over the whole duration of the direct signal, giving reduced distortion. 

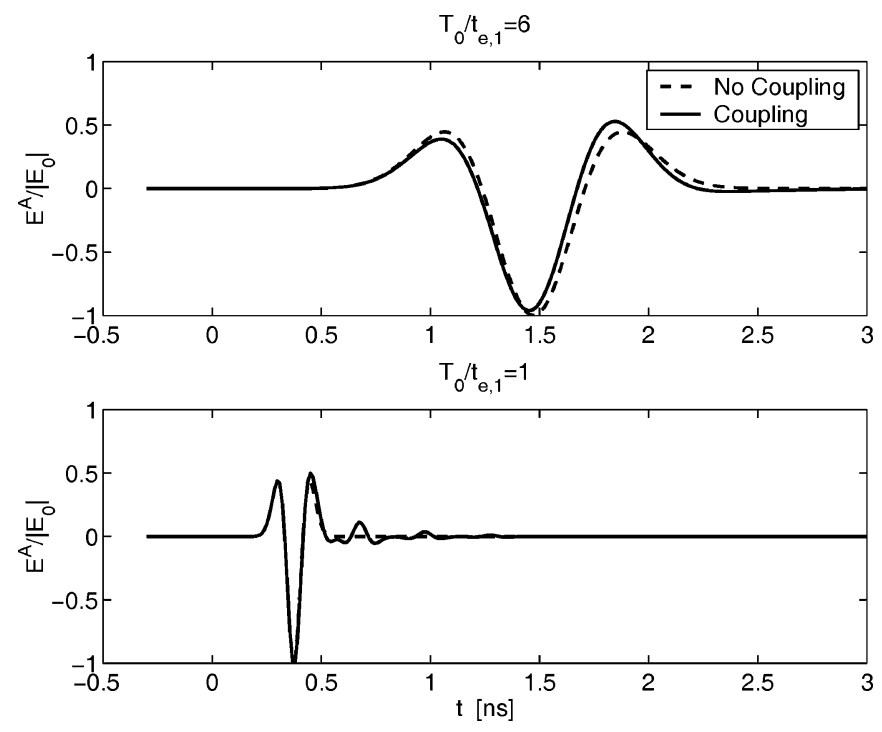

Fig. 9. Radiated field along the main beam (at $\theta_{0}=30^{\circ}$ ) for an $N=4$ element array with and without the inclusion of the coupling effects, for two different durations $T_{0}$ of the gaussian pulse.
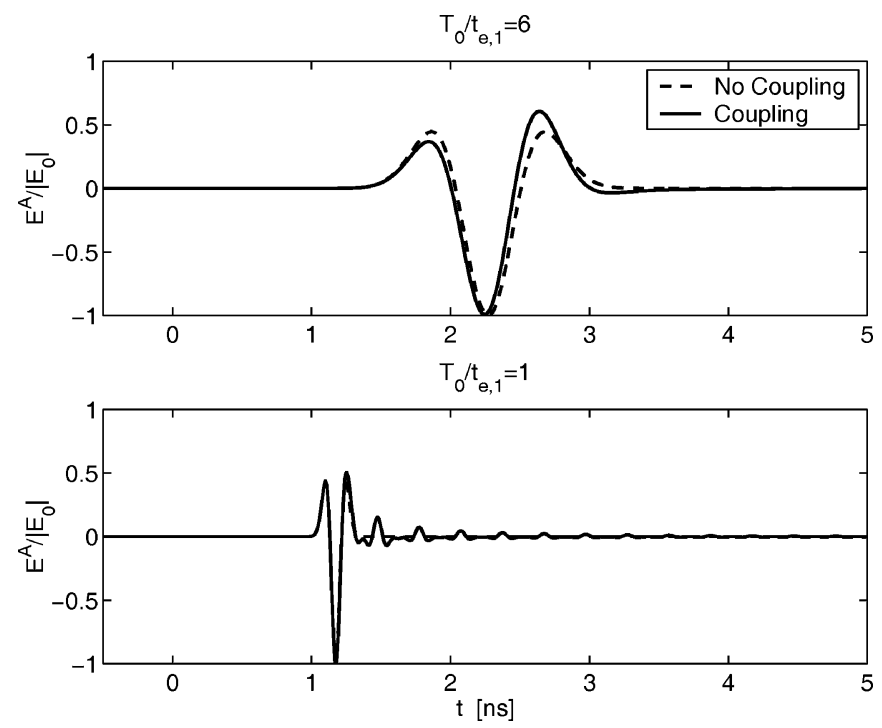

Fig. 10. Radiated field along the main beam (at $\theta_{0}=30^{\circ}$ ) for an array of $N=20$ elements with and without the inclusion of the coupling effects, for two different gaussian pulse durations $T_{0}$.

Mutual coupling always affects the signal in the endfire direction, quite apart from the signal duration.

To compare the effect of coupling for two different durations of the driving signals, Fig. 9 shows the field radiated by an array of $N=4$ isotropic radiating elements with main beam at $\theta_{0}=30^{\circ}$. It can be observed that (Fig. 9, bottom) for small values of $T_{0} / t_{e, 1}$ (short-duration signals), the main part of the radiated signal is not sensibly distorted (Fig. 9, bottom) since the echoes appear delayed with respect to the direct pulse. As $T_{0} / t_{e, 1}$ increases the resulting signal is more distorted (Fig. 9, up) since the echoes approach the main part of the direct signal.

If a larger array is considered, for instance having $N=20$ elements (Fig. 10) a greater distortion is apparent in the main wavepacket of the radiated signal for long-duration pulses. Instead, only a longer ringing is produced in the late transient in

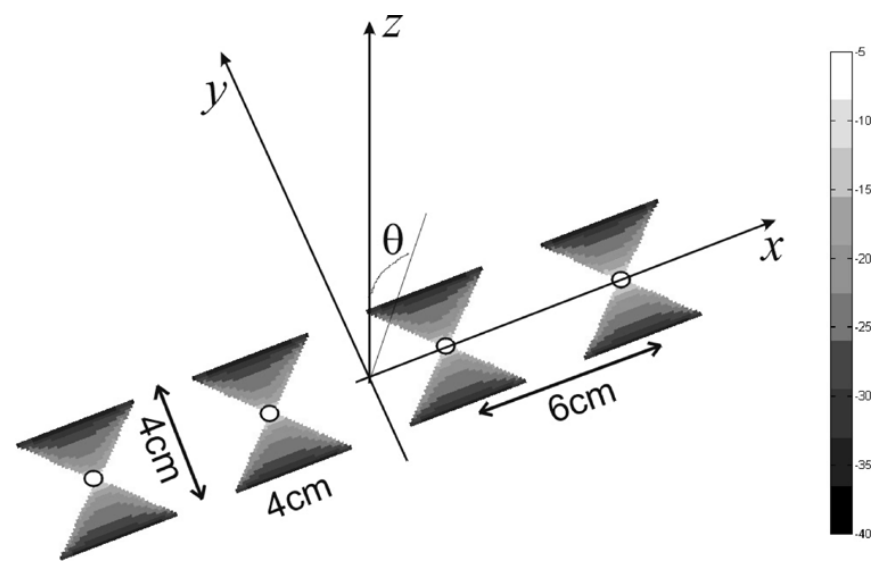

Fig. 11. Linear array of four bow-ties. Antennas are sourced in the gap. The shadowed pattern is the energy norm of the induced currents used for the Radon transform.
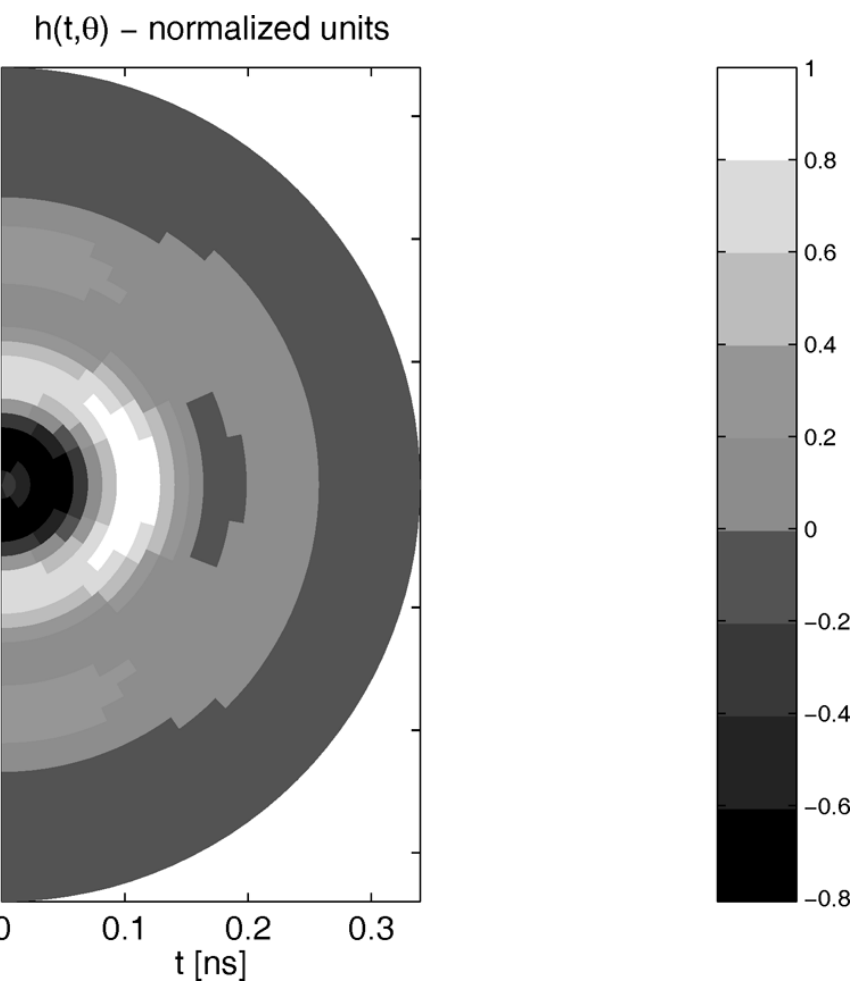

Fig. 12. Fullwave-computed time-domain effective height in $(t-\theta)$ plane for the standalone bow-tie.

the case of very short input pulses (Fig. 10, bottom) since each oscillation belongs to different element echoes.

For real arrays, the antenna effective height and antenna-generator mismatch play an important role on the signal distortion due to coupling. The generator mismatch causes a distortion of the radiated signals, with a broadening of its duration. This effect is however taken into account in both the antenna effective height (which includes the antenna-generator mismatch) and in the expression of the active array factor through the embedded reflection operators $\gamma_{L, n}(\tau)$ applying to incoming current echoes. As a consequence, the signal distortion in the array will be relevant for narrow-band and unmatched antennas since 

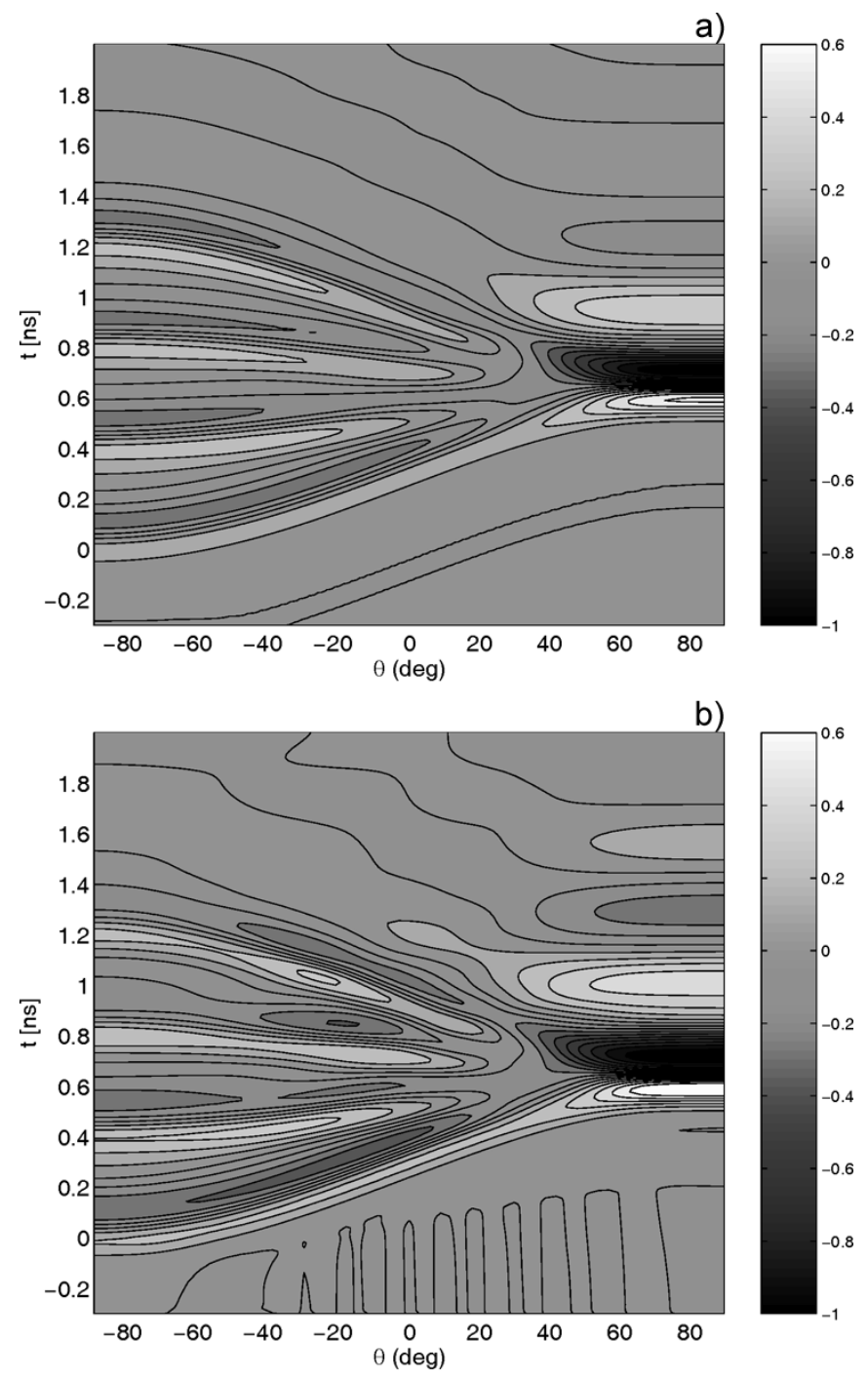

)
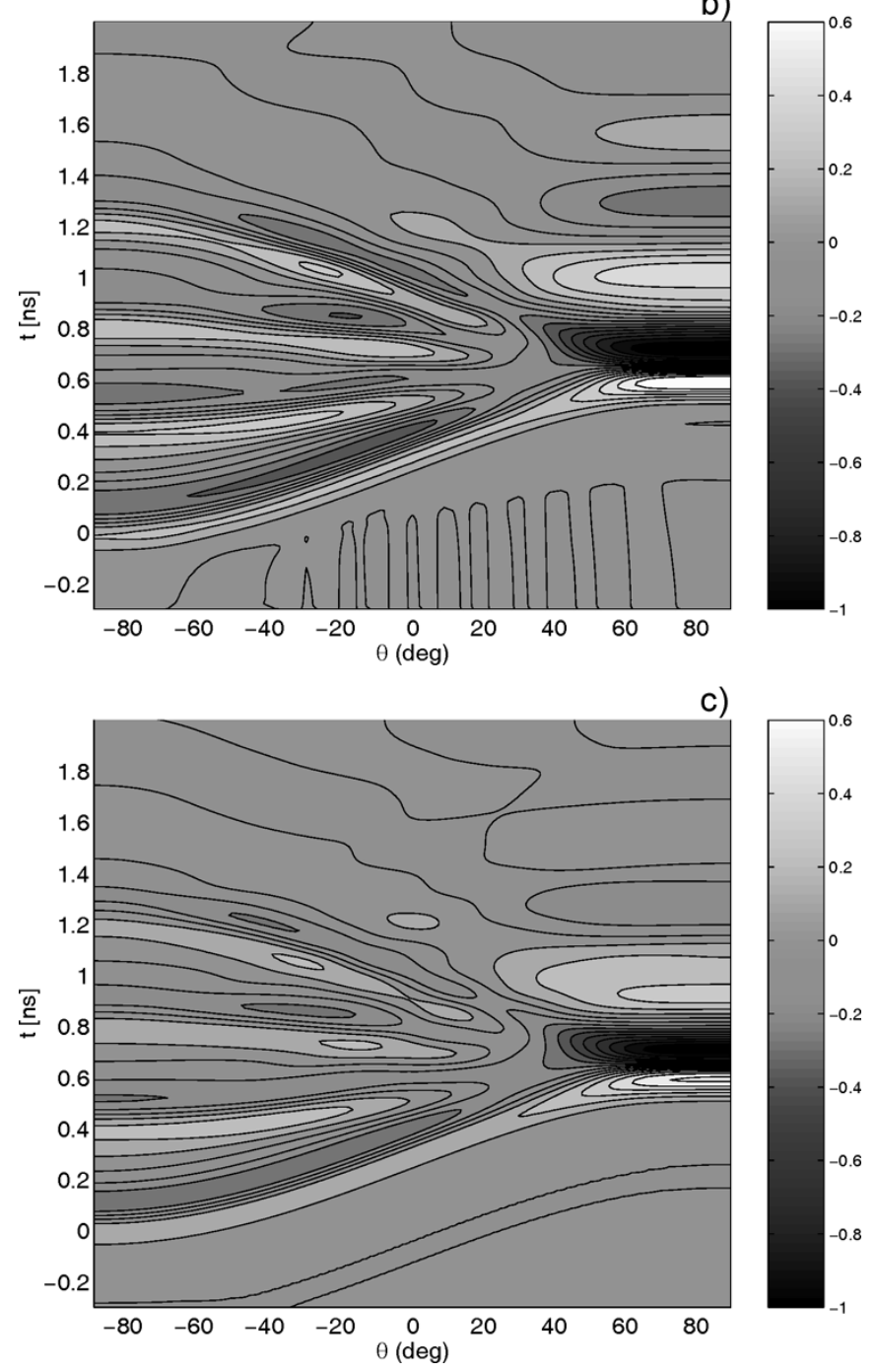

Fig. 13. Bow-tie array sourced by $T_{0}=0.3 \mathrm{~ns}$ gaussian pulses timed to produce endfire focusing: (a) array field neglecting coupling; (b) fullwave array field including coupling; (c) approximated array field with coupling computed by the active array factor in (6) with $G_{0}=0.15$.

the broad coupling echoes will be easily overlapped to the direct signal.
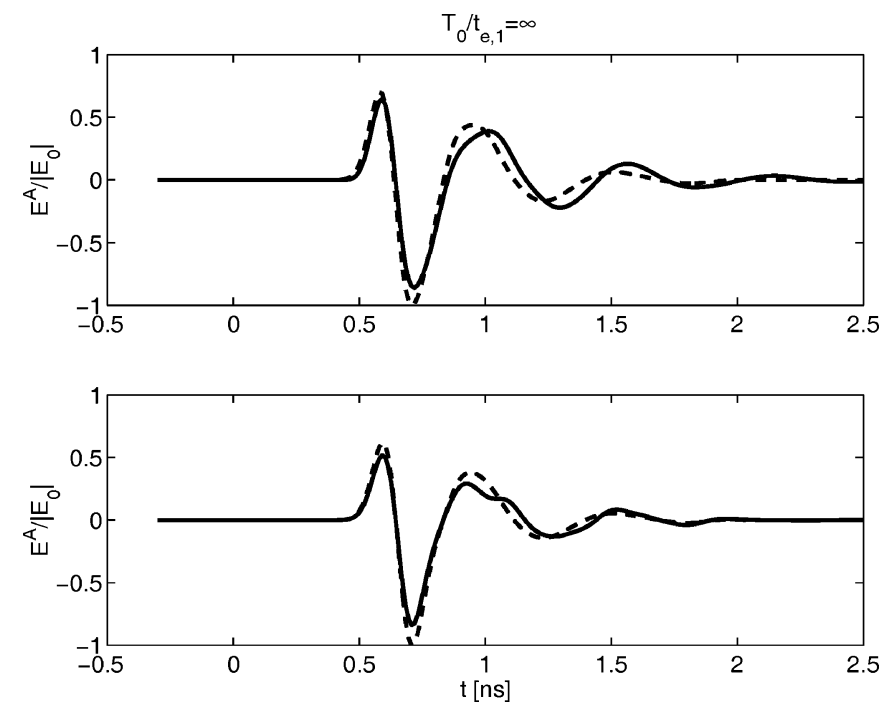

Fig. 14. Cuts of the transient field in Fig. 13 at $\theta=90^{\circ}$, comparing the array response with (continuous lines) and without coupling (dashed lines) for the fullwave simulation (up) and for the simplified model (down).
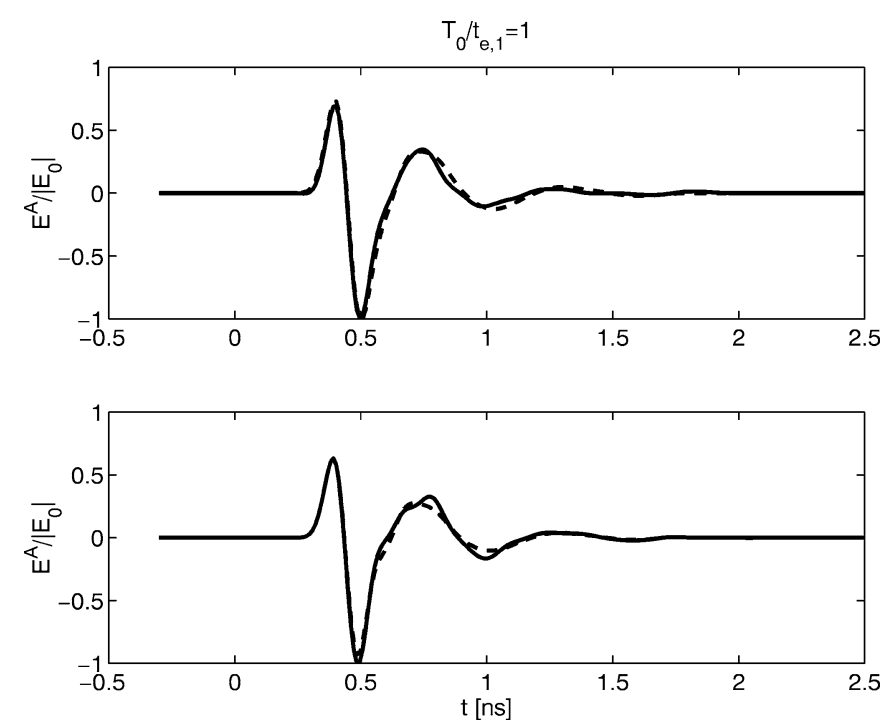

Fig. 15. Radiated field for an $N=4$ bow-tie array along the main beam at $\theta_{0}=30^{\circ}$ with (continuous line) and without (dashed lines) the inclusion of the coupling effects, for the fullwave simulation (up) and for the model (down).

\section{A FullWAVE ExAMPLE}

The coupling models introduced in previous sections are now employed to discuss the radiation from a realistic linear array of $N=4$ square bow-tie antennas of $4 \mathrm{~cm}$ side and $6 \mathrm{~cm}$-spaced (Fig. 11). The antennas are fed in the gap by a voltage generator having $50 \Omega$ internal resistance and producing a gaussian pulse, $v_{0}(t)$, of duration $T_{0}$.

The structure has been modeled by the finite-difference timedomain method [9], [10]. The time-variant surface current density $\boldsymbol{J}(\rho, t)=\hat{n} \times \boldsymbol{H}_{0}(\rho, t), \boldsymbol{H}_{0}$ being the time-dependent magnetic field evaluated on the array conductors, has been then used 
to calculate the radiated far field via the application of the Radon transform [6]

$$
\boldsymbol{r} \boldsymbol{E}^{\boldsymbol{A}}(\boldsymbol{r}, \tau)=-\frac{\mu}{4 \pi} \frac{\partial}{\partial t} \int \boldsymbol{J}_{\|}\left(\boldsymbol{\rho}, \tau+\frac{\hat{\boldsymbol{r}} \cdot \boldsymbol{\rho}}{c}\right) d S
$$

with $\boldsymbol{J}_{\|}=\boldsymbol{J}-\hat{\boldsymbol{r}}(\hat{\boldsymbol{r}} \cdot \boldsymbol{J})$. Numerical results therefore account for all the coupling mechanisms among antennas. The field obtained is compared with that predicted as convolution between the time-domain coupling-free array factor and the TD effective height of the isolated bow-tie (Fig. 12). Additionally, it is also shown the transient field obtained as $\mathcal{F}_{A} * \boldsymbol{h}^{t} * v_{0}(t)$ where the simplified expression of the active array factor in (6) and the fullwave computed effective height in Fig. 12 are used. More in detail, having observed that the average reflection coefficient of the standalone bow-tie in the $2-10 \mathrm{GHz}$ band is $-8 \mathrm{~dB}$ and that the average coupling factor among contiguous elements is of the order of $-8.5 \mathrm{~dB}$, the global factor $G_{0}=S_{0} \Gamma_{0} / d$ in (6) has been set to $G_{0}=0.15$. Accordingly, Fig. 13 shows the space-time patterns, obtained by the different models, when the input signal $v_{0}(t)$ has a duration $T_{0}=0.3 \mathrm{~ns}$ and the array is timed to produce end-fire radiation.

It can be possible to observe that the effect of the time-domain coupling appears, in both the fullwave model [Fig. 13(b)] and in the simplified one [Fig. 13(c)], as a broadening of the third oscillation in the endfire direction and as additional oscillations along side lobes for $\theta<0$.

Cuts in Fig. 14 of the transient fields in Fig. 13 at $\theta=30^{\circ}$ show a good agreement between the fullwave results and the simplified models, in particular for what concerns the $20 \%$ reduction of the signal peak due to interference of coupling echoes. Finally, Fig. 15 shows the radiated field when the array is focused at $\theta_{0}=30^{\circ}$. For this case the input signal $v_{0}(t)$ has short duration compared with the time delay of first echo $\left(T_{0} / t_{e 1}=1\right)$ and, as expected from the qualitative analysis in the previous Section, the distortion is nearly negligible since the echoes begin to overlap the direct signal after the third oscillation.

\section{CONCLUSION}

The phenomenology and the effects of ultrawide-band array coupling have been investigated through the definition of general expressions for the time-domain active array factor and active element factor. Under some further simplifications, the interaction among multiple radiators has been described, in the case of short pulse, as superposition of multiple echoes, scattered by the antennas and superimposed to the direct signals, therefore producing distortion. The strength of the coupling depends not only on the inter-element spacing and on the scan angle, as in the frequency domain, but also on the antenna bandwidth and on transmitted waveforms. In particular, the distortion is more relevant for long-duration signals with fast repetition rate, and also increases with the reduction of the antenna bandwidth. Depending on the above parameters, the effect of the coupling consists in a change of the main part of the direct signal or more often in the addition of extra-ringing in the signal tail.

The time-domain models proposed in this paper have yielded some useful relationships among the geometrical parameters (array spacing), the system bandwidth (e.g. the duration and repetition rate of the transmitted signal) and the scan angle, which could be considered in order to reduce the coupling even in the case of very dense arrays. The most potentially risky configuration occurs in the endfire radiation since some coupling echoes are fully synchronized with the direct pulses and distortions are visible also for short-duration signals. However, the coupling phenomena in UWB arrays are rather modest and much less relevant than in the frequency domain since coupling echoes generally appear at different times and therefore the overall summation is less effective than in the case of continuous signals (monochromatic arrays). Moreover, UWB arrays can be also used in sparse configurations, and in these cases the coupling effects are really negligible.

The proposed model has been discussed for 1-D arrays but it can be easily extended to two-dimensional configurations.

\section{REFERENCES}

[1] M. G. M. Hussain, "Principles of space-time array processing for ultrawide-band impulse radar and radio communications," IEEE Trans. Veh. Technol., vol. 51, no. 3, pp. 393-403, 2002.

[2] A. Shlivinski and E. Heyman, "A unified kinematic theory of transient arrays," in Ultra-Wideband Short-Pulse Electromagnetics 5, P. D. Smith and S. R. Cloude, Eds. New York: Kluwer Academic/Plenum Publisher, 2000, pp. 327-334.

[3] L. D. DiDomenico, "A comparison of time versus frequency domain antenna patterns," IEEE Trans. Antennas Propag., vol. 50, no. 11, pp. 1560-1566, 2002.

[4] J. L. Schwartz and B. D. Steinberg, "Ultrasparse, ultrawideband arrays," IEEE Trans. Ultrason., Ferroelectr. Freq. Control, vol. 45, no. 2, pp. 376-393, 1998.

[5] D. M. Pozar, "The active element pattern," IEEE Trans. Antennas Propag., vol. 42, no. 8, pp. 1176-1178, 1994.

[6] A. Shlivinski, E. Heyman, and R. Kastner, "Antenna characterization in the time domain," IEEE Trans. Antennas Propag., vol. 45, no. 7, pp. $1140-1149,1997$.

[7] G. Marrocco and F. Capolino, "Transient radiation by periodic structures: accuracy of the (Time Domain-Floquet Wave)-FDTD algorithm," in Proc. IEEE Antennas and Propagation Society Symp., vol. 3, San Antonio, TX, 2002, pp. 643-646.

[8] _ _ "A combined Floquet-Wave-FDTD algorithm for the modeling of transient radiation from infinite periodic structures," in Electromagnetics in a Complex World: Challenges and Perspectives. Berlin, Germany: Springer-Verlag, 2004, pp. 249-257.

[9] A. Taflove, Computational Electrodynamics: The Finite Difference Time Domain Method. Norwood, MA: Artech House, 1995.

[10] G. Marrocco and F. Bardati, "BEST: A finite-difference solver for time electromagnetics," Simulation Practice Theory, vol. 7, pp. 279-293, Jul. 1999. 


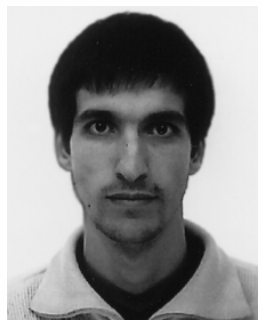

Matteo Ciattaglia (S'04) received the Laurea degree in telecommunication engineering at the University of Rome "Tor Vergata," Rome, Italy, in 2002, where he is currently working toward the Ph.D. degree.

In 2003 he was Grant Researcher at the University of Rome "Tor Vergata", working on numerical methods for time-domain electromagnetics. He is currently employed at SELEX SI, S.p.A. (formerly Alenia Marconi Systems, S.p.A.), Rome, Italy, working on phased array modeling. His research interests are in the area of time domain electromagnetics, UWB technology, numerical modeling, optimization methods.

Mr. Ciattaglia received the "Mario Sannino" award for the Best Scientific Presentation at the National Italian Congress of Electromagnetism (XV RiNEm) in 2004.

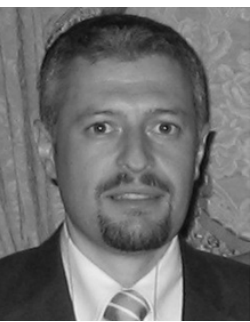

Gaetano Marrocco received the Laurea degree in electronic engineering and the Ph.D. degree in applied electromagnetics from the University of L'Aquila, L'Aquila, Italy, in 1994 and 1998, respectively

Since 1997, he has been a Researcher at the University of Rome "Tor Vergata," Rome, Italy, where he currently teaches antenna design and bioelectromagnetics. In summer 1994, he was at the University of Illinois at Urbana-Champain as Postgraduate Student. In autumn 1999, he was a Visiting Researcher at the Imperial College in London, London, U.K. He has been involved in several space, avionic and naval programs of the European Space Agency, NATO, Italian Space Agency, and the Italian Navy. His research is mainly directed to the development of numerical methods and signal processing techniques for the time domain modeling and design of complex electromagnetic structures in the context of biological and aerospace applications. 